\title{
Geometrically induced singular behavior of entanglement
}

\author{
D. Cavalcanti, ${ }^{1}$ P. L. Saldanha, ${ }^{2}$ O. Cosme, ${ }^{2}$ F. G. S. L. Brandão, ${ }^{3,4}$ C. H. Monken, ${ }^{2}$ S. Pádua, ${ }^{2}$ \\ M. França Santos, ${ }^{2}$ and M. O. Terra Cunha ${ }^{5}$ \\ ${ }^{1}$ ICFO-Institut de Ciencies Fotoniques, Mediterranean Technology Park, 08860 Castelldefels, Barcelona, Spain \\ ${ }^{2}$ Departamento de Física, Universidade Federal de Minas Gerais, Caixa Postal 702, 30123-970, Belo Horizonte, Minas Gerais, Brazil \\ ${ }^{3}$ QOLS, Blackett Laboratory, Imperial College London, London SW7 2BW, United Kingdom \\ ${ }^{4}$ Institute for Mathematical Sciences, Imperial College London, London SW7 2BW, United Kingdom \\ ${ }^{5}$ Departamento de Matemática, Universidade Federal de Minas Gerais, Caixa Postal 702, 30123-970, Belo Horizonte, \\ Minas Gerais, Brazil
}

(Received 31 August 2007; published 10 July 2008)

\begin{abstract}
We show that the geometry of the set of quantum states plays a crucial role in the behavior of entanglement in different physical systems. More specifically, it is shown that singular points at the border of the set of unentangled states originate singularities in the dynamics of entanglement of smoothly varying quantum states. We illustrate this result by implementing a photonic parametric down-conversion experiment. Moreover, this effect is connected to recently discovered singularities in condensed matter models.
\end{abstract}

DOI: 10.1103/PhysRevA.78.012318

PACS number(s): 03.67.Mn, 02.40.Pc, 03.65.Ud

Entanglement, a genuine quantum correlation, plays a crucial role in different physical situations ranging from information processing [1] to quantum many-particle phenomena [2]. As in thermodynamics, smooth variations of controllable parameters which characterize a physical system may lead to singular behavior of entanglement quantifiers. In some cases, in similarity to quantum phase transitions [3], these singularities are attested by abrupt changes in the quantum state describing the system. However, unexpected singularities may appear even when the quantum state varies smoothly [4]. Here we demonstrate how the geometry of the set of unentangled states can be related to singular behavior in physical phenomena. In particular, we show that singularities at the boundary of this set can be detected by measuring the amount of entanglement of smoothly varying quantum states.

Entangled states are defined as the states of composed quantum systems which cannot be written as a convex sum of products of the density matrices for each composing part [5]. Separable states, on the other hand, admit such a representation and form a convex, closed set with positive volume (for finite-dimensional systems) [6]. This set, henceforth designated by $S$, is a subset of $D$, the set of all density matrices $(S \subset D)$, which is also convex and closed. Therefore, a natural geometric way to quantify entanglement is to see how far-using some definition of distance on the state space-an entangled state is from the set $S$. This has been carried over for a variety of notions of distance, generating different measures of entanglement [7]. One of these geometric quantifiers is the random robustness $R_{R}$, defined for any state $\rho$ as the minimum $s(s \geq 0)$ such that the state

$$
\sigma=\frac{\rho+s I / d}{1+s}
$$

is separable, where $I$ is the identity matrix and $d$ the total dimension of the state space [8]. The physical motivation is clear: $\sigma$ represents a mixture of $\rho$ with the random state $I / d$, and $R_{R}(\rho)$ quantifies how much of this noise must be added to $\rho$ in order to obtain a separable state.

Our main result is to show that $R_{R}$ can be used to investigate the shape of the boundary of $S, \partial S$. The principle is to take an entangled state depending smoothly on one parameter $q$ and compute $R_{R}$ as a function of $q$. The one-parameterdependent density matrices $\rho(q)$ can be seen as a curve in the set of quantum states as shown in Fig. 1. Singularities at $\partial S$ will show up as singularities in $R_{R}(\rho(q))$. This statement is general for any finite dimension and will be formalized by the contrapositive: if $\partial S$ is nonsingular, then $R_{R}(\rho(q))$ is also nonsingular. This result will be formally proven in the Appendix and we emphasize its interpretation: any singularity in $R_{R}$ for a well-chosen path $\rho(q)$ reflects singularities in $\partial S$.

Let us now focus on the case for two qubits, which is related to the experiment described here. In this case, Ref. [10] shows that the random robustness is proportional to the negativity $(\mathcal{N}(\rho))[11]$, given by the absolute value of the sum of the negative eigenvalues of the partially transposed state. The negativity is a monotone under local operations and classical communication [12] and has the operational interpretation of a cost function under a certain class of operations $[13,14]$.

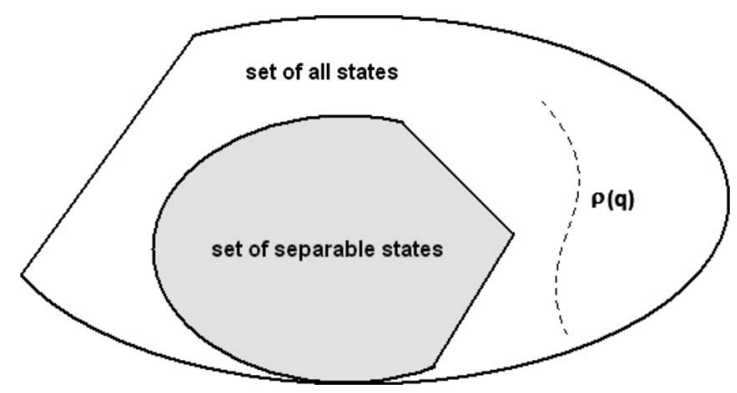

FIG. 1. State space. The dotted line represents the path $\rho(q)$ followed by $\rho$ when the parameter $q$ is changed. It is worth noting that $S$ can present singular points in its shape and to remember that the "true" picture is much subtler, given the large dimensionality of even the simplest example [9]. 


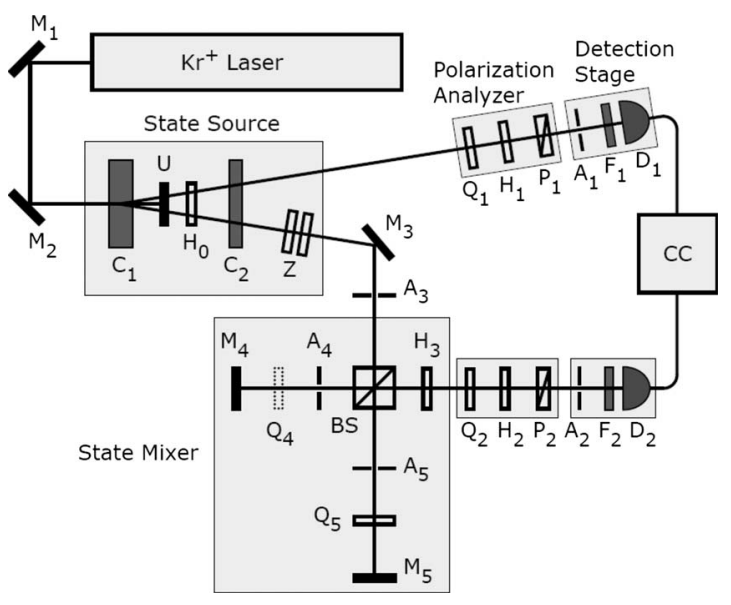

FIG. 2. Experimental setup: The state source is composed by a 2-mm-thick $\left(\beta-\mathrm{BaB}_{2} \mathrm{O}_{4}\right)(\mathrm{BBO})$ nonlinear crystal $\left(C_{1}\right)$ pumped by a cw krypton laser operating at $413 \mathrm{~nm}$, generating photon pairs at $826 \mathrm{~nm}$ by type-II spontaneous parametric down-conversion. Crystal $C_{1}$ is cut and oriented to generate either one of the polarizationentangled Bell states $\left|\Psi_{-}\right\rangle$or $\left|\Psi_{+}\right\rangle$. Walk-off and phase compensation is provided by the half-wave plate $H_{0}$ followed by a $1-\mathrm{mm}$ thick $\mathrm{BBO}$ crystal $\left(C_{2}\right)$ [23], together with two 1-mm-thick crystalline quartz plates $(z)$ inserted in one of the down-converted photon paths. The unconverted laser beam transmitted by crystal $C_{1}$ is discarded by means of a dichroic mirror $(u)$. The detection stages are composed by photon counting diode modules $D_{1}$ and $D_{2}$, preceded by $8 \mathrm{~nm}$ full width at half maximum interference filters $F_{1}$ and $F_{2}$ centered at $825 \mathrm{~nm}$, and by circular apertures $A_{1}$ of $1.6 \mathrm{~mm} \varnothing$ and $A_{2}$ of $3.0 \mathrm{~mm} \varnothing$. Single and coincidence counts with 5 ns resolving time are registered by a computer-controlled electronic module (CC). Polarization analyzers are composed of quarter-wave plates $Q_{1}$ and $Q_{2}$, half-wave plates $H_{1}$ and $H_{2}$, followed by polarizing cubes $P_{1}$ and $P_{2}$. The state source produces state $\left|\Psi_{-}\right\rangle$. For each pair, the photon emerging in the upper path goes straight to the polarization analyzer and to the detection stage 1. The lower-path photon is directed by mirror $M_{3}$ through the circular aperture $A_{3}$ into the state mixer (an unbalanced Michelson interferometer), composed by the beam splitter BS, mirrors $M_{4}$ and $M_{5}$, quarter-wave plates $Q_{4}$ and $Q_{5}$, variable circular apertures $A_{4}$ and $A_{5}$, and by the half-wave plate $H_{3}$, whose purpose is to compensate for an unwanted slight polarization rotation caused by the beam splitter. The quarter-wave plate $Q_{4}$ is switched off, which means that if the lower photon follows the path labeled 4 , there is no change to its polarization and the half-wave plate $H_{3}$ changes the state to $\left|\Psi_{+}\right\rangle$. On the other hand, if the lower photon follows the path labeled 5, $Q_{5}$ is oriented with the fast axis at $45^{\circ}$ in order to flip its polarization. The path length difference, $130 \mathrm{~mm}$, is much larger than the coherence length of the down-converted fields, ensuring an incoherent recombination at BS. The pair detected by $\mathrm{CC}$ is in state $q\left|\Psi_{+}\right\rangle\left\langle\Psi_{+}|+(1-q)| \Phi_{+}\right\rangle\left\langle\Phi_{+}\right|$, where $q$ is defined by the relative sizes of apertures $A_{4}$ and $A_{5}$.

At the same time, entanglement can be measured with the help of entanglement witnesses [15]. These are Hermitian operators with positive mean value for all separable states, but with a negative mean value for some entangled states [16]. In fact, many geometrical entanglement quantifiers are directly related to witness operators [17]. In the particular case of two qubits, ${ }^{1}$ we have that for every entangled state $\rho$ [10],

$$
2 \mathcal{N}(\rho)=R_{R}(\rho)=-2 \min _{W \in \mathcal{W}} \operatorname{Tr}(W \rho),
$$

where $\mathcal{W}$ is the set of entanglement witnesses $W$ with $\operatorname{Tr} W=2$.

At this point we might ask some natural questions. Is there in fact any singularity in the shape of $S$ ? In the affirmative case, does this singularity appear in any physical setup? We proceed to answer both questions positively by showing physical processes where a singularity in $\partial S$ is revealed by monitoring the entanglement of a given system.

First, let us consider a general system of four qubits $a, b$, $A$, and $B$, subject to the following Hamiltonian [19]:

$$
H=H^{a A}+H^{b B}
$$

where

$$
H^{\mu \nu}=\frac{\omega}{2} \sigma_{z}^{\mu}+\frac{\omega}{2} \sigma_{z}^{\nu}+\frac{g}{2}\left(\sigma_{-}^{\mu} \sigma_{+}^{\nu}+\sigma_{+}^{\mu} \sigma_{-}^{\nu}\right)
$$

Here $\sigma_{+}=\left(\sigma_{x}+i \sigma_{y}\right) / 2$ and $\sigma_{-}=\left(\sigma_{x}-i \sigma_{y}\right) / 2$, where $\sigma_{x}, \sigma_{y}$, and $\sigma_{z}$ are the usual Pauli matrices. This scenario can be realized in many systems, like cavity QED [20], trapped ions [21], and quantum dots [22]. We set the initial state to be $|\psi(t=0)\rangle=\left|\Phi_{+}\right\rangle_{a b} \otimes\left|\Psi_{+}\right\rangle_{A B}$, where the qubits $a b$ are in the Bell state $\left|\Phi_{+}\right\rangle=(|00\rangle+|11\rangle) / \sqrt{2}$ and the qubits $A B$ are in the orthogonal Bell state $\left|\Psi_{+}\right\rangle=(|01\rangle+|10\rangle) / \sqrt{2}$. The Hamiltonian (3) induces a swapping process which leads (in the interaction picture) to the following temporal evolution for the subsystem $A B$, obtained by tracing out the subsystem $a b$ :

$$
\rho_{A B}(t)=q\left|\Psi_{+}\right\rangle\left\langle\Psi_{+}|+(1-q)| \Phi_{+}\right\rangle\left\langle\Phi_{+}\right|,
$$

where $q=\cos ^{2}(g t)$. For this state the negativity reads

$$
\mathcal{N}\left(\rho_{A B}(t)\right)=\max \{1-2 q, 2 q-1\}=|1-2 q| .
$$

This function presents a singularity for $q=0.5(\mathrm{~g} t=n \pi / 4$, with $n$ odd) signaling then a singularity at $\partial S$.

Another physical process that also produces the family of states (5) is the following simple quantum communication task. Alice prepares a Bell state $\left|\Phi_{+}\right\rangle$and sends one qubit to Bob through a quantum channel; if this channel has a probability $q$ of introducing a bit flip, and $1-q$ of no error at all, the state (5) is the output of the process. ${ }^{2}$

\footnotetext{
${ }^{1}$ An optimal entanglement witness $W_{\text {opt }}$ satisfying (2) is proportional to the partial transposition of the projector over the eigenspace of the negative eigenvalue of $\rho^{T_{2}}$, where $\rho^{T_{2}}$ denotes the partial transposition of $\rho$ [18].

${ }^{2}$ The simplest way of drawing the complete line represented by Eq. (5) is to consider three different initial conditions: from $\left|\Phi_{+}\right\rangle$ one obtains $q \in[0,1 / 2)$, from $\left|\Psi_{+}\right\rangle, q \in(1 / 2,1]$, and $q=1 / 2$ is a fixed point of this dynamical system.
} 

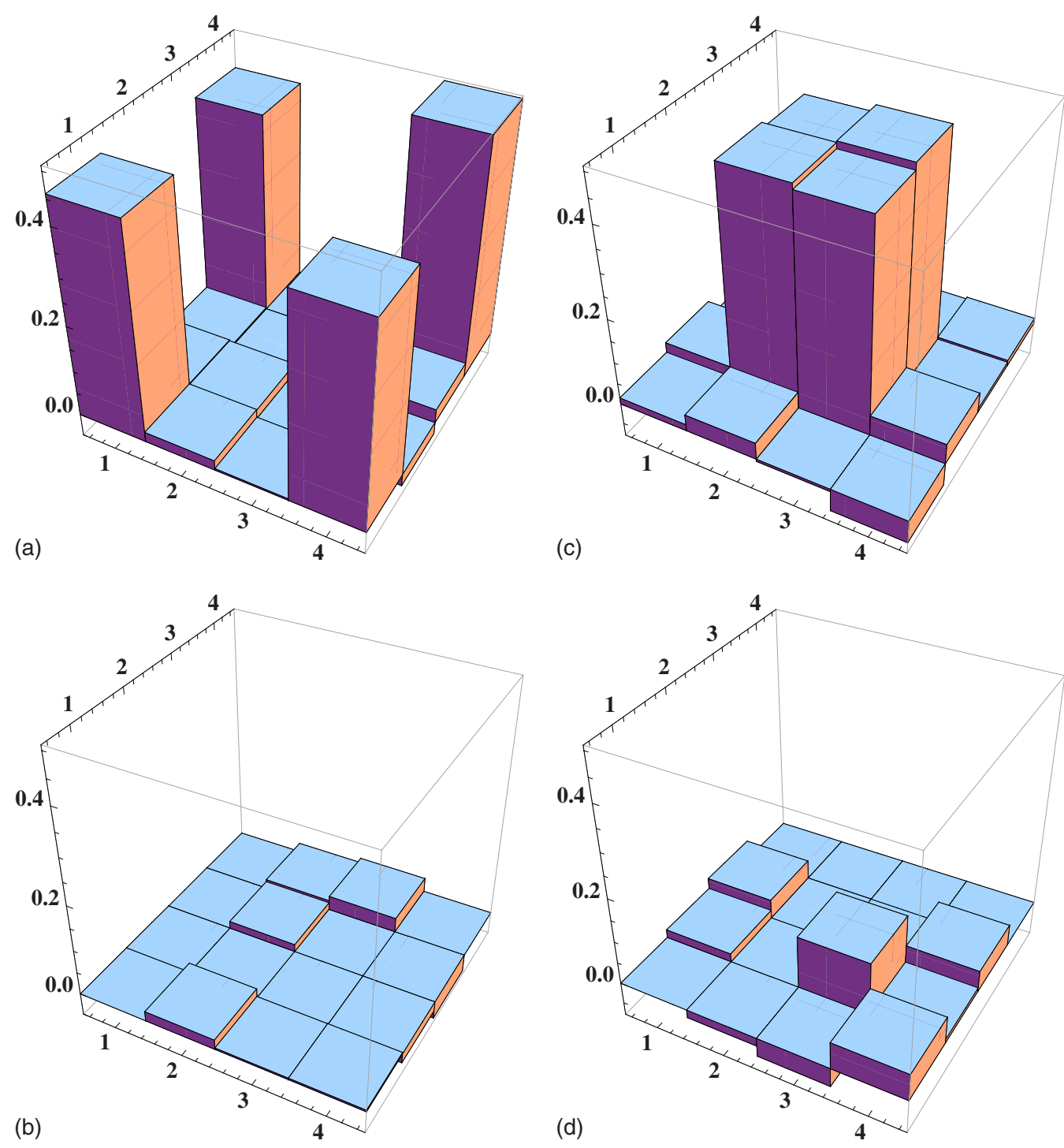

FIG. 3. (Color online) Reconstructed density matrices corresponding (ideally) to the states $\left|\Phi_{+}\right\rangle$, (a) real and (b) imaginary parts, and $\left|\Psi_{+}\right\rangle$, (c) real and (d) imaginary parts. The attained fidelities for these states are, respectively, $F_{\Phi_{+}} \equiv\left\langle\Phi_{+}|\rho| \Phi_{+}\right\rangle \approx(92 \pm 4) \%$ and $F_{\Psi_{+}}$ $\equiv\left\langle\Psi_{+}|\rho| \Psi_{+}\right\rangle \approx(96 \pm 4) \%$.

To illustrate the dynamics given by Eq. (5), we have performed an optical experiment, shown in Fig. 2. In our experiment, twin photons maximally entangled in polarization are generated in a nonlinear crystal [23] and sent to an unbalanced Michelson interferometer, which is used to simulate the channel described above. The experiment works as follows. We produce a two-photon $\left|\Psi_{+}\right\rangle$state, and send one of the photons directly to the detection stage and the other to the (unbalanced) interferometer. One of the arms of this interferometer does not change the polarization of the photon, and if the photon went through this path the two photons would be detected in $\left|\Psi_{+}\right\rangle$. However, if the photon went through the other path its polarization would be rotated in such a way that the final two-photon state would become $\left|\Phi_{+}\right\rangle$. We have made a tomographic characterization of the photonic states corresponding to these two extremal points. The reconstructed density matrices are displayed in Fig. 3. These two possibilities are then incoherently recombined, thus allowing the preparation of state (5). The parameter $q$ is controllable, and the experiment is repeated for different values of $q$. The corresponding optimal witness is given by

$$
W_{\mathrm{opt}}=\left\{\begin{array}{l}
I-2\left|\Phi_{+}\right\rangle\left\langle\Phi_{+}\right| \text {for } 0 \leq q \leq 1 / 2, \\
I-2\left|\Psi_{+}\right\rangle\left\langle\Psi_{+}\right| \text {for } 1 / 2 \leq q \leq 1 .
\end{array}\right.
$$

For the family of generated states these two observables are the only candidates for optimal entanglement witnesses, so they are the only ones to be measured. In a more general situation, if less is known about the prepared state, many more candidate witnesses should be measured. The results are displayed in Fig. 4. The blue (continuous) curve in the figure shows the witnessed negativity measurement and its edge indicates the existence of singularities at $\partial S$. This experimental result shows the abrupt change in the optimal witness at the value $q=\frac{1}{2}$, which heralds the singularity in $\partial S$. As a proof of principles, each operator $W$ is measured for the 


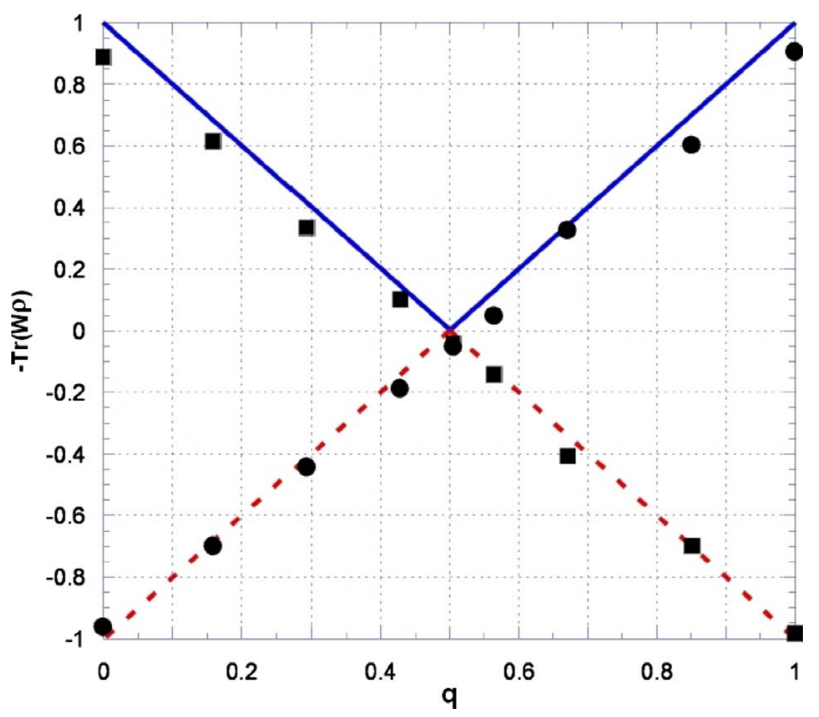

FIG. 4. (Color online) Measurement of the mean value of both operators described in (7) for the full range $0 \leq q \leq 1$. Each $W$ is expanded as a linear combination of products of local operators which are then measured independently. The blue continuous line corresponds to the theoretical value of $\mathcal{N}(\rho(q))$ for the state $\rho(q)$ $=q\left|\Psi_{+}\right\rangle\left\langle\Psi_{+}|+(1-q)| \Phi_{+}\right\rangle\left\langle\Phi_{+}\right|$. Note that each $W$ witnesses entanglement for only a restricted range of $q$ values as predicted by the theory. The local singularity of $\partial S$ is evidenced by the abrupt change of optimal $W$. Experimental errors are within the dots' sizes.

whole range of $q$, which yields the points below zero in Fig. 4. Note that the singularity occurs exactly for $R_{R}=0(q$ $=1 / 2$ ). According to our geometrical interpretation, this means that the path followed by the parametrized state $\rho(q)$ touches the border of $S$. This result should not be a surprise, since it is well known that in the tetrahedron generated by the Bell states (which we access in our experiment) the separable states form an inscribed octahedron [24]. It must be stressed that in higher dimensions geometrically induced singularities can occur at nonzero entanglement values.

The geometrical properties of entanglement discussed here give new insight into singularities found recently in the entanglement of condensed matter systems. Striking examples, dealing with entanglement properties of certain spin- $\frac{1}{2}$ models subjected to a transverse magnetic field $h$, are described in Refs. [4]. In these works, the two-qubit reduced state shows a singularity in entanglement for a particular field value $h_{f}$ far from the critical field of the corresponding model. As the correlation functions, ground state energy, and even reduced density matrices are all smooth at $h_{f}$, there was no clear origin for these singularities. Our results offer an explanation by interpreting the nonanalyticities exhibited by entanglement as a consequence of geometric singularities at $\partial S^{3}$

As previously mentioned, $R_{R}$ can be used to probe $\partial S$ in

\footnotetext{
${ }^{3}$ Although the results of Refs. [4] were obtained in terms of the concurrence, a completely analogous result holds for the negativity as well
}

any finite-dimensional system. For example, a previous work showed a singular behavior of $R_{R}$ in three-qubit systems [10]. Within the scope of our paper, we can interpret it as originated by a singularity at the border of the corresponding separable set. Note, however, that in this case, due to the higher dimensionality of the system, the singularity at $\partial S$ manifests itself far away, at a point with positive random robustness.

To sum up, we have presented the consequences found of the shape of the set of separable states. Singularities in this set were found and connected to nonanalytical behavior of entanglement in different physical systems. It is an interesting open question to find physical implications of such singularities.

We acknowledge discussions with A. Acín, A. Sen(De), J. Wehr, E. Rico, G. Palacios, V. Vedral, and J. Dunningham and funding from CNPq, Fapemig, PRPq-UFMG, and the Brazilian Millenium Institute for Quantum Information.

\section{APPENDIX}

Here we formally prove that $R_{R}$ can detect singularities in $\partial S$. We state it as a general proposition for convex sets, but the reader will naturally recognize the context.

Proposition 1. Let $D$ be a closed, convex set. Let $S \subset D$ also be closed and convex, with $\pi$ a point in the interior of $S$. If $\partial S$ is a $C^{m}$ manifold and the states $\rho(q)$ describe a $C^{m}$ curve in $D$ with no points in the interior of $S$ and obeying the condition that the tangent vector $\rho^{\prime}(q)$ is never parallel to $\pi-\rho(q)$, then $R_{R}(\rho(q))$ is also a $C^{m}$ function.

A manifold is called $C^{m}$ if it can be parametrized by functions with continuous derivatives up to order $m$ [25]. The reader can change $C^{m}$ to $C^{\infty}$, with almost no loss (actually, we choose $C^{m}$ as being "as regular as necessary"). Other topological remarks before the proof: the fact that $S$ has interior points implies that $S$ and $D$ have the same dimensionality (since there is an open ball of $D$ contained in $S$ ), and the proof will use the notion of the (topological) cone, which simply means the union of all segments from a given point $V$ to each point of a given set $A$; this is called the cone of $A$ with vertex $V$.

Proof. The geometrical situation leads to the cone given by $(p, q) \mapsto p \pi+(1-p) \rho(q), p \in[0,1]$. The condition on the tangent vector [together with the fact that $\pi$ is interior to $S$, while $\rho(q)$ has no point in this interior] is sufficient for this cone to be $C^{m}$, except at the vertex $\pi$, at least locally in $q$.

As $S$ is bounded and convex, and $\pi$ is in its interior, every straight line from $\pi$ crosses $\partial S$ exactly once. As $\rho(q)$ has no point in the interior of $S$, this crossing always happens for $0 \leq p<1$. Denote this crossing value by $p_{c}(q)$. The curve $q \mapsto p_{c}(q) \pi+\left[1-p_{c}(q)\right] \rho(q)$ is $C^{m}$, implying that $p_{c}$ is a $C^{m}$ function of $q$.

The random robustness is given by $R_{R}(\rho(q))=\frac{p_{c}}{1-p_{c}}$. As $p_{c}<1$, we also obtain that $R_{R}$ is a $C^{m}$ function of $q$. 
[1] A. K. Ekert, Phys. Rev. Lett. 67, 661 (1991).

[2] L. Amico, R. Fazio, A. Osterloh, and V. Vedral, Rev. Mod. Phys. 80, 517 (2008).

[3] T. J. Osborne and M. A. Nielsen, Phys. Rev. A 66, 032110 (2002); A. Osterloh, L. Amico, G. Falci, and R. Fazio, Nature (London) 416, 608 (2002).

[4] A. Osterloh, G. Palacios, and S. Montangero, Phys. Rev. Lett. 97, 257201 (2006); T. Roscilde, P. Verrucchi, A. Fubini, S. Haas, and V. Tognetti, ibid. 93, 167203 (2004); T. Roscilde, P. Verrucchi, A. Fubini, S. Haas, and V. Tognetti, ibid. 94, 147208 (2005).

[5] R. F. Werner, Phys. Rev. A 40, 4277 (1989).

[6] K. Życzkowski, P. Horodecki, A. Sanpera, and M. Lewenstein, Phys. Rev. A 58, 883 (1998).

[7] R. Horodecki, P. Horodecki, M. Horodecki, and K. Horodecki, arXiv:quant-ph/0702225; M. B. Plenio and S. Virmani, Quantum Inf. Comput. 7, 1 (2007).

[8] G. Vidal and R. Tarrach, Phys. Rev. A 59, 141 (1999).

[9] I. Bengtsson and K. Życzkowski, Geometry of Quantum States: An Introduction to Quantum Entanglement (Cambridge University Press, Cambridge, U.K., 2006).

[10] F. G. S. L. Brandão and R. O. Vianna, Int. J. Quantum Inf. 4, 331 (2006).

[11] G. Vidal and R. F. Werner, Phys. Rev. A 65, 032314 (2002). Note that in our work the negativity for two qubits runs from 0 to 1 .

[12] J. Eisert, Ph.D. thesis, University of Potsdam, 2001, e-print arXiv:quant-ph/0610253.

[13] K. Audenaert, M. B. Plenio, and J. Eisert, Phys. Rev. Lett. 90, 027901 (2003).

[14] S. Ishizaka, Phys. Rev. A 69, 020301(R) (2004).
[15] M. Barbieri, F. DeMartini, G. DiNepi, P. Mataloni, G. M. D'Ariano, and C. Macchiavello, Phys. Rev. Lett. 91, 227901 (2003); O. Gühne et al., J. Mod. Opt. 50, 1079 (2003); M. Bourennane, M. Eibl, C. Kurtsiefer, S. Gaertner, H. Weinfurter, O. Guhne, P. Hyllus, D. Bruss, M. Lewenstein, and A. Sanpera, Phys. Rev. Lett. 92, 087902 (2004); D. Cavalcanti and M. O. Terra Cunha, Appl. Phys. Lett. 89, 084102 (2006); K. Audenaert and M. B. Plenio, New J. Phys. 8, 266 (2006); O. Gühne, M. Reimpell, and R. F. Werner, Phys. Rev. Lett. 98, 110502 (2007); J. Eisert, F. G. S. L. Brandão, and K. M. R. Audenaert, New J. Phys. 9, 46 (2007).

[16] M. Horodecki, P. Horodecki, and R. Horodecki, Phys. Lett. A 223, 1 (1996); B. M. Terhal, ibid. 271, 319 (2000).

[17] F. G. S. L. Brandão, Phys. Rev. A 72, 022310 (2005).

[18] M. Lewenstein, B. Kraus, J. I. Cirac, and P. Horodecki, Phys. Rev. A 62, 052310 (2000).

[19] D. Cavalcanti, J. G. Oliveira Jr, J. G. Peixoto de Faria, M. O. Terra Cunha, and M. F. Santos, Phys. Rev. A 74, 042328 (2006).

[20] J. M. Raimond, M. Brune, and S. Haroche, Rev. Mod. Phys. 73, 565 (2001).

[21] M. D. Barrett et al., Nature (London) 429, 737 (2004).

[22] W. D. Oliver, F. Yamaguchi, and Y. Yamamoto, Phys. Rev. Lett. 88, 037901 (2002).

[23] P. G. Kwiat, K. Mattle, H. Weinfurter, A. Zeilinger, A. V. Sergienko, and Y. Shih, Phys. Rev. Lett. 75, 4337 (1995).

[24] R. Horodecki and M. Horodecki, Phys. Rev. A 54, 1838 (1996).

[25] M. Spivak, Calculus on Manifolds: A Modern Approach to Classical Theorems of Advanced Calculus (W.A. Benjamin, New York, 1965). 